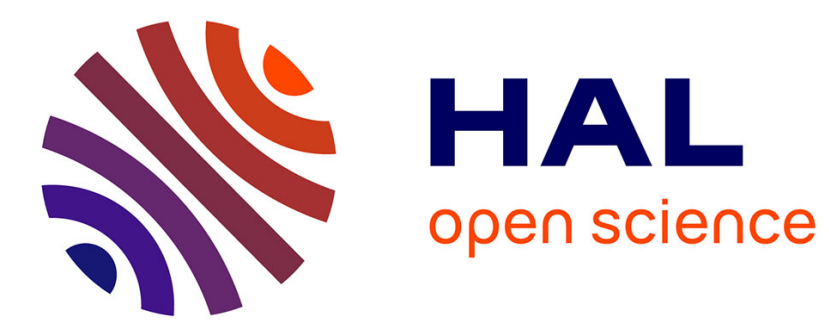

\title{
Etudes expérimentales sur l'enseignement à distance
} Philippe Dessus, Benoît Lemaire, Jacques Baillé

\section{To cite this version:}

Philippe Dessus, Benoît Lemaire, Jacques Baillé. Etudes expérimentales sur l'enseignement à distance. Sciences et Techniques Educatives, 1997, 4 (2), pp.137-164. 10.3406/stice.1997.1338 . hal-01297171

\section{HAL Id: hal-01297171 https://hal.science/hal-01297171}

Submitted on 3 Apr 2016

HAL is a multi-disciplinary open access archive for the deposit and dissemination of scientific research documents, whether they are published or not. The documents may come from teaching and research institutions in France or abroad, or from public or private research centers.
L'archive ouverte pluridisciplinaire HAL, est destinée au dépôt et à la diffusion de documents scientifiques de niveau recherche, publiés ou non, émanant des établissements d'enseignement et de recherche français ou étrangers, des laboratoires publics ou privés. 


\title{
Études expérimentales sur l'enseignement à distance
}

\section{Experimental studies on distance teaching and learning}

\author{
Philippe Dessus - Benoît Lemaire - Jacques Baillé \\ DEACT, Laboratoire des Sciences de l'Éducation \\ Université Pierre-Mendès-France \\ BP 47 \\ 38040 GRENOBLE CEDEX \\ E-mail :Prénom.Nom@upmf-grenoble.fr
}

\begin{abstract}
RÉSUMÉ. Les nombreuses études sur la communication et l'enseignement à distance ont plus été centrées sur le programme que sur la théorie ou l'expérimentation contrôlée. Les chercheurs se sont longtemps plus préoccupés de concevoir de nouveaux produits plutôt que d'évaluer, sur un plan théorique et/ou expérimental, l'efficacité de ces derniers. Nous commençons par analyser la manière dont sont traitées, dans le champ de l'enseignement à distance, les théories sur l'apprentissage, l'enseignement, la conception de séquences d'enseignement et la communication. Nous recensons ensuite une quinzaine d'études expérimentales. Ces études, issues des champs de recherche suivants : $\mathrm{CMC}, \mathrm{CSCW}, \mathrm{CHI}$ et $\mathrm{EAD}$, mesurent les effets comparatifs de divers facteurs et sont regroupées selon la tâche des sujets : communication interpersonnelle, production de document ou de procédure et enfin suivi de cours. Les principaux résultats de ces études ne nous permettent pas de poser que l'utilisation de nouveaux médias opère une influence significative sur la communication ou l'apprentissage.
\end{abstract}

\begin{abstract}
Studies on distance learning and communication are mostly based on the program rather than on theories or experiments. Researchers are more interested in designing new products than in evaluating on a theoretical and/or experimental basis their efficiency. First, we analyze the way the theories on learning, teaching, instructional design and communication are handled in the field of distance learning. Then, we describe about fifteen experimental studies in the field of $\mathrm{CMC}, \mathrm{CSCW}, \mathrm{CHI}$ and distance learning. Their goal is to measure the comparative effects of various factors. They are grouped together according to the subjects' tasks : communicating with other people, designing a procedure or a document, following a course. The major result of these studies is that using new medias does not have a significant effect on communication or learning.
\end{abstract}

MOTS-CLÉS : Enseignement à distance (EAD), média, communication, face à face, visioconférence, Computer-mediated communication (CMC), Computer-supported cooperative work (CSCW), Computer-human interaction (CHI). 
KEYWORDS: Distance learning, media, communication, face-to-face, videoconference, computer-mediated communication (CMC), computer-supported cooperative work (CSCW), computer-human interaction (CHI).

\begin{abstract}
"Récemment, certaines Universités introduisirent les circuits télé, puis les cassettes préenregistrées qui permettaient de gratter au son de la voix doctorale. Les étudiants ne constatant aucune différence avec la présence physique du docteur, l'expérience fut interrompue, démontrant trop à l'évidence l'inutilité des cours. "Rien ne remplace la présence physique des enseignants", glapirent-ils! Tu parles! Tout montrait qu'il revenait parfaitement au même que le perroquet soit vivant ou filmé. Il pérorait toujours. »

B. MARIS (1991). Les sept péchés capitaux des universitaires. Paris : Albin Michel, p. 77-78.
\end{abstract}

\title{
1. Introduction
}

L'enseignement à distance (désormais EAD) bénéficie d'une image ambiguë, il jouit, en tant que «nouvelle technique d'éducation» d'une image favorable, dans l'air du temps, que renvoient opportunément les nombreuses études basées sur la satisfaction des élèves - voir, parmi les plus récentes, [D'AM 94 ; FUL 93 ; KIE, 96 ; SAG 95], où les étudiants jugent qu'il est plus aisé d'étudier avec un nouveau média qu'en face à face avec un enseignant - et pâtit d'un préjugé néfaste, conséquence du précédent : cet a priori positif n'encourage guère les chercheurs à valider expérimentalement les effets d'un EAD. Nous retombons ici dans un schéma classique, déjà avéré à propos de l'enseignement assisté par ordinateur : le seul fait de travailler « à la pointe » efface les critiques et évaluations. La recherche en EAD, comme l'expriment [SAB 94], se centre sur les programmes plutôt que sur la théorie. Il importe plus de justifier, a posteriori, l'utilisation d'un nouveau système technique que de l'asseoir a priori, à partir d'une théorie. La recherche en EAD, entre sciences de l'information et de la communication et sciences de l'éducation, 
opère une fuite en avant, avec de nouveaux programmes qui cachent mal leur faible assise théorique et/ou méthodologique.

Il nous semble utile de reprendre les principes théoriques qui étayent les recherches expérimentales en EAD et de les vérifier ensuite chez certaines de ces dernières. Les chercheurs travaillant sur ce sujet sont face à deux dilemmes : (1) reprend-on les principes théoriques de l'enseignement en face à face ou peut-on s'en démarquer ? (2) quel champ de recherche explorer ? En effet, si le nombre d'études effectuées sur l'EAD est important, certains travaux dans des domaines voisins tels que l'interaction homme-machine ou la communication pourraient être transposables à un contexte d'enseignement. Comme l'EAD utilise des médias ${ }^{1}$ - d'ailleurs de plus en plus diversifiés - , doit-on donc scruter les différences liées à l'utilisation de ces médias ? Si la communication élèves-enseignant et élèvesélèves est changée, quels principes théoriques sont à même d'en rendre compte ? Si les processus d'enseignement - planification, choix de méthodes - et d'apprentissage sont également modifiés dans un contexte d'EAD, de quelle manière le sont-ils ? Essayons maintenant d'apporter quelques éléments de réponse à ces questions.

\section{Quelques théories sur l'enseignement à distance}

Nous avons repéré quelques thèmes récurrents discutés dans les problématiques sur l'EAD. Nous les listons ci-dessous en essayant de montrer en quoi ils sont bénéfiques à l'avancée de la recherche expérimentale. À cette fin, la classification de [WAG 94] est fort utile pour repérer ce à quoi ont trait les différentes théories concurrentes : elle distingue les théories de l'apprentissage (learning theories), les théories de l'enseignement (instructional theories), la conception de séquences

\footnotetext{
${ }^{1}$ Nous avons opté pour l'orthographe en vigueur du mot « média », plutôt que pour la forme anglo-saxonne.
} 
d'enseignement (instructional design) et enfin la transmission (instructional delivery). C'est cette classification que nous employons maintenant.

\subsection{Théories de l'apprentissage : $d u$ « chalk and talk » au constructivisme}

[WEB 95] note que, à l'ère du tout numérique, le média le plus couramment

utilisé est encore la craie et la parole (the chalk and talk). Des chercheurs travaillant dans le domaine de la communication par ordinateur (computer-mediated communication) et du travail collaboratif informatisé (computer-supported collaborative work) ont tenté de proposer une utilisation du matériel plus conforme à la théorie constructiviste et à son extension pour l'apprentissage, l' « apprentissage en situation » (situated learning). [JON 94] proposent ainsi des repères pour le passage d'un raisonnement sur les symboles à un apprentissage en situation. La construction d'une connaissance ne peut se passer d'une composante sociale et, de plus, ne peut être étudiée en laboratoire, décontextualisée. Des dispositifs techniques comme les logiciels de courrier et de communication électroniques et certains logiciels de type hypertexte ou de simulation rassemblent beaucoup d'avantages pour étudier l'apprentissage en situation: ils favorisent et enregistrent les transactions. Toutes ces nouvelles formes d'apprentissage semblent idéales dans leur application à l'enseignement à distance : elles utilisent un média électronique, favorisent le contrôle de l'enseignement par les élèves ainsi que les groupements de travail. Mais ne nous y trompons pas, quel que soit le média choisi, et l'EAD ne fait pas exception, les symboles ne sont pas évacués car une partie de l'apprentissage des élèves est de prendre connaissance du «monde de symboles » du média utilisé [MAY 88].

Les données sur l'apprentissage des élèves sont souvent inférées de l'étude de leur comportement. Or on trouve peu d'éléments concernant ce problème, la plupart des études «à distance » portant sur une situation de communication de type « réunion d'affaires ». Pourtant, la spécificité du contexte (absence d'enseignant) fait que ce paramètre pourrait distinguer notablement la situation «à distance » d'une situation « en présence », au moins en ce qui concerne la «présence sociale » 
de l'enseignant [SHO 76, cités par SEL 95]. Et quand les études se préoccupent de l'apprenant, c'est pour se centrer sur des facteurs à notre sens annexes, comme leur satisfaction. C'est moins l'apprentissage qui est invoqué que des aspects techniques ou de contenu, mais faiblement reliés à l'expérience réelle des apprenants. Voyons maintenant ce qu'il en est de l'enseignant.

\subsection{Théories de l'enseignement : de la distance de transaction à l'analyse du discours de l'enseignant}

La distance de transaction (transactional distance), établie par [MOO 93], permet, à l'aide de trois paramètres que sont la structure, le dialogue et l'autonomie de l'élève, de rendre compte de la distance séparant l'enseignant des élèves. La structure «exprime la rigidité ou la flexibilité des objectifs pédagogiques du programme, des stratégies d'enseignement et des méthodes d'évaluation ». Elle décrit «dans quelle mesure un programme d'enseignement peut s'adapter aux besoins personnels de chaque apprenant» (id., p. 26). Le dialogue «se manifeste dans le flot des interactions enseignant-élève, lorsque l'un donne une instruction et que les autres répondent » (id., p. 23-24). La troisième dimension, l'autonomie de l'élève, définit la possibilité de ce dernier à déterminer lui-même plutôt que l'enseignant des variables comme les buts, méthodes et modalités d'évaluation. [MOO 93] insiste sur le fait que la distance de transaction est une variable continue plutôt que discrète, un enseignement en face à face comportera également une certaine distance de transaction et il s'agit, dans l'idéal, de trouver une distance maximisant le dialogue et l'autonomie de l'élève tout en minimisant la structure (voir figure 1). Les logiciels de type tutoriel ou communication par ordinateur minimisent ainsi la distance de transaction, accrue pour des dispositifs de type radiophonique, livre programmé ou bien enseignement assisté par ordinateur. 


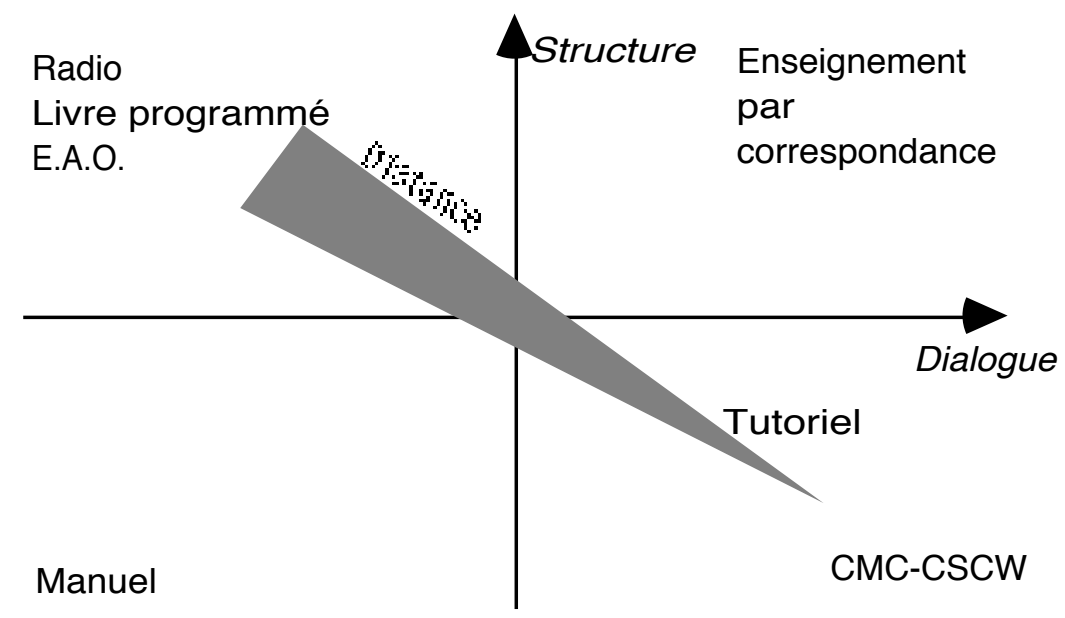

Figure 1. Distance de transaction et quelques médias, d'après [M00 93]. La distance de transaction est maximale lorsqu'on minimise le dialogue tout en rigidifiant la structure, et vice versa.

Il importe de souligner, avec [JAC 93], que cette distance enseignant-élève n'est pas fortuite, mais qu'elle est le «fondement de la relation pédagogique» (p. 56). Cette distance, qui pourrait être considérée comme un handicap à la bonne diffusion d'un enseignement, est érigée en principe. Dans la plupart des cas, les effets (positifs ou négatifs) d'un enseignement à distance sont attribués à cette même distance, alors que l'expérimentation contrôle mal les effets annexes de la situation, qui peuvent, eux aussi, rejaillir sur les résultats des élèves. Cela énoncé, il est tout aussi clair que n'importe quel enseignant, qu'il soit en situation d'EAD ou non, est amené à « apprivoiser la distance », selon l'expression de [JAC 93] : les devoirs à la maison, les interrogations, les (auto-)évaluations, le travail collaboratif sont autant de moyens de pallier cette distance enseignant-élève. Les distances du tableau à la chaise de l'élève, de l'enseignant au domicile de l'élève sont autant de distances que 
l'enseignant essaie de réduire ou, du moins, d'apprivoiser ${ }^{2}$. De plus, l'enseignant n'est pas la seule personne à laquelle se réfèrent les élèves, l'EAD nécessite une équipe complète de techniciens et de facilitateurs qui opèrent à l'interface enseignant-élèves, ce qui peut également augmenter la distance subjective entre ces derniers.

L'analyse du discours de l'enseignant, depuis [SIN 75], a été l'objet d'études importantes - voir [CAZ 86], pour une revue de la question. Il est trivial de dire que l'EAD place ses protagonistes dans une situation de communication, mais nous devons essayer de comprendre ce qu'elle a de spécifique, notamment dans ses caractéristiques implicites [CON 96]. Décrivons rapidement deux méthodes d'analyse qui nous semblent prometteuses en ce qu'elles sont faciles à mettre en œuvre et relativement indépendantes du contenu du discours de l'enseignant, la méthode de Bronckart [BRO 85], centrée sur les caractéristiques morphosyntaxiques et la méthode de Henri [HEN 91], centrée sur la pragmatique du discours. La méthode de Bronckart se situe au niveau morphosyntaxique et permet de caractériser la condition de production d'un discours en la référant à des situations architypiques (narration, en situation, théorique). Il peut être intéressant d'observer si les nouvelles conditions de production que créent les nouvelles technologies affectent les discours correspondants. En particulier, que devient un discours en situation lorsque s'intercale un dispositif technique entre les participants ? [LEM 96]. [HEN 91] signale que la connaissance que nous pouvons avoir des échanges à distance ${ }^{3}$ enseignant-élèves est assez superficielle (nombre et durée des tours de parole). Elle préconise l'utilisation de méthodes d'analyse du discours plus attachées au sens. La théorie des actes de langage de Searle [SEA 82]

2 On peut se reporter à l'intéressante étude de [ULB 95] qui tente de diminuer encore la distance de transaction en augmentant, via un simple dispositif de commande, le feed-back des élèves vers l'enseignant.

3 L'auteur pense aux échanges via un ordinateur, mais cela nous semble généralisable à tout type de média, comme nous le laissent penser les études centrées sur le processus décrites infra (voir tableau I). 
peut nous aider à aller dans ce sens, en classant les « points illocutoires », actes de langage mis en ouvre dans une situation de communication en cinq catégories : assertif, directif, expressif, engageant et déclaratif.

Nous avons jusqu'à présent parlé de théories sur l'enseignement en laissant de côté un champ qui tend de plus en plus à l'autonomie, celui de l'instructional design, que nous traduirons (mal) par « conception de séquences d'enseignement ». Ce champ, qui est très étudié dans la littérature anglo-saxonne, tente de proposer des modèles théoriques qui globalisent l'activité de l'enseignant (avant, pendant et après l'enseignement).

\subsection{La conception de séquences d'enseignement à distance}

La tâche de concevoir un cours à distance a été reconnue, par certains, comme la partie la plus critique liée à la réussite des élèves [WHI 87, cité par JOH 91]. Dans un contexte de face à face, concevoir un cours est déjà un processus complexe, à propos duquel il reste de nombreuses zones d'ombre [TOC 93]. En EAD, le travail est encore plus difficile, du fait, notamment, de l'autonomie de l'apprenant, qui se forge beaucoup plus librement son parcours de formation. L'enseignant n'est donc plus seulement un «programmeur», un communicant, mais aussi un facilitateur d'apprentissage et doit apporter un soin particulier au séquencement des cours [SHE 94] . L'élève, lui, se place de ce fait en concepteur d'une partie du cours. En face à face, le problème est le rapport enseignant-élève : une augmentation du nombre des élèves entraînant plus de matériel, de salles, d'enseignants, ce qui n'est pas le cas en EAD, où le problème et donc le coût majeur est la conception de supports de cours [DEW 94].

La conception de séquences d'EAD a été encore peu étudiée, en grande partie parce que la plupart des modèles généraux considèrent l'utilisation d'un média

particulier comme un problème annexe, qui influe peu sur le processus global d'enseignement. Les modèles à venir devront sans doute prendre davantage en 
compte l'influence du média, non pas seulement sur l'apprentissage, où elle est controversée, mais sur le processus d'enseignement/apprentissage dans sa totalité.

\subsection{Les médias : interaction, interactivité et transmission des connaissances}

Commençons par une définition: les médias sont les «moyens basés sur la technologie, permettant de diffuser et de conserver des informations ainsi que de développer des attitudes, de susciter une rétroaction et de favoriser l'expression » [LEG 93]. En tant que « technologie », le média est donc au centre de l'attention des apprenants et enseignants, puisqu'il est censé véhiculer l'enseignement pour les premiers et être l'objet de décisions institutionnelles chez les seconds.

Le terme d'interaction se retrouve très souvent dans la littérature sur l'ordinateur « média d'enseignement ». L'interaction homme-machine fait partie depuis longtemps des thèmes majeurs de la théorie sur les nouvelles techniques éducatives. Est-ce que le fait de parler d'EAD change l'acception de ce terme ? D'après [WAG 94], il semble que oui. En effet, elle propose de distinguer «interaction » interpersonnelle, attribut de l'enseignement effectif, d' «interactivité », reliée au système qui médiatise l'enseignement. Ainsi, nous pouvons voir que ce terme d'interaction est bien spécifique à l'EAD, par exemple de type « visioconférence ».

[WOL 95] met en avant quatre principales différences entre enseignement en présence et EAD : la distance interpersonnelle accrue, la réduction du nombre et de la fréquence des interactions, l'absence de feed-back et une compréhension des messages diminuée du fait de l'absence d'un canal visuel. Les trois dernières différences sont reliées à l'interaction, ce qui souligne bien l'importance de ce facteur. L'interaction correspond, chez la plupart des auteurs, à ce que [MOO 93] appelle le dialogue, même si ce dernier fait une distinction entre les deux termes en donnant à ce dernier une connotation positive ( «Un dialogue est réfléchi, constructif et apprécié de chaque interlocuteur »id., p. 24).

L'EAD, en tant que média d'enseignement, peut être ajouté au célèbre débat entre Clark et Kozma à propos de l'effet du média sur l'apprentissage des élèves. Il 
serait trop long de décrire en détail cette discussion étalée sur une décennie (voir, dans l'ordre chronologique, [CLA 83 ; KOZ 91 ; CLA 94 ; KOZ 94 ; CAR 96]), qui d'ailleurs a eu peu d'écho dans la littérature française. Il s'agit de vérifier l'influence directe des médias sur l'apprentissage. Pour [CLA 83] les médias sont les épiceries du savoir, mais n'ont pas d'influence directe sur l'alimentation (apprentissage) des élèves. [KOZ 91 ; KOZ 94], lui, pense qu'il faut s'attacher à trouver des situations où le média puisse, dans le futur, influencer l'apprentissage, même s'il a tendance à rejoindre Clark sur la non-efficacité actuelle des médias sur l'apprentissage.

Ces deux positions se retrouvent dans la littérature à propos des médias. La première fait des recherches sur les médias, en essayant d'isoler la variable «média », la deuxième avec les médias, en proposant des situations complexes, certes plus écologiques, mais dans lesquelles la variable «média» peut se confondre avec d'autres variables, comme la méthode d'exposition des connaissances. De très nombreuses recherches ont été menées suivant la première des pistes, outre les auteurs déjà cités, [JOH 91] et [RUS 95] ont réalisé sur ce thème de bonnes revues de la question. Une des idées discutées par [JOH 91] est que l'efficacité d'un média doit être évaluée par une mesure plus large que celle de résultats à des tests : la motivation des élèves, l'engagement des parents, la communication entre structures éducatives, etc. [RUS 95], quant à lui, ne recense pas moins de deux cent dix-huit résultats de recherche concluant à une différence intermédias non significative.

Il convient d'être vigilant, les coûts financiers et humains résultant de la mise en place de programmes d'EAD peuvent être plus importants que les retombées effectives en termes d'apprentissage et de facilité d'enseignement. Sans aller jusqu'à l'ironie de [STE 88, cité par BRE 96] disant qu' «il y a plus de gens qui font de la recherche sur le télétravail que de télétravailleurs eux-mêmes », ironie qui ne nous semble pas transposable mot pour mot à l'EAD, il n'est pas certain que ce dernier soit à tout prix la meilleure voie. L'EAD doit-il s'imposer systématiquement? Quels contextes sont les plus favorables ? Alors que le monde de l'entreprise commence à prendre la mesure du télétravail [BRE 96], qu'en est-il 
dans le monde de l'éducation, et surtout que devrait-il en être si l'on considère les résultats effectifs de ce type d'enseignement?

\section{3. Études expérimentales}

\subsection{Délimitation du champ de recherche}

Essayons maintenant d'ébaucher quelques pistes bibliographiques à propos de recherches expérimentales en EAD. Cet article va donc être orienté par les tâches et activités des protagonistes d'une situation d'enseignement/apprentissage, plutôt que par une vue historique, souvent préférée dans la littérature française [GLI 91 ; JAC 93]. Nous avons passé en revue, pour ce faire, des ouvrages, revues et actes de colloques dans les domaines de recherche suivants : CMC (computer-mediated communication), CSCW (computer-supported cooperative work), HCI (computerhuman interaction) et EAD.

Définissons tout d'abord brièvement chacun de ces domaines de recherche :

Le CMC : « [...] est le seul média permettant les téléconférences qui soit asynchrone. Il fonctionne comme un courrier électronique, communiquant du texte écrit numérique. Il requiert une liaison entre les terminaux, afin de les utiliser en réseau, ce dernier pouvant être local, national ou international » [LAU 93].

Avec [DIX 94], nous pouvons considérer que le CMC peut être utilisé pour réaliser un travail collaboratif ou coopératif ${ }^{4}$. Toutefois, un courant récent émerge, le $\mathrm{CSCW}$, permettant à plusieurs opérateurs de coopérer ou de collaborer, dans une situation de travail, autour d'un système technique informatisé [DEM 95].

4 La différence entre un travail coopératif et un travail collaboratif est expliquée par [HEN 96]. Le premier entraîne une spécialisation des opérateurs en vue de réaliser la tâche, alors que le deuxième fait travailler les opérateurs en parallèle, sans véritable spécialisation ou distribution des tâches à accomplir [DIL s.d.]. 
[JOH 92] donne une définition générale du HCI, qui nous conviendra ici, c'est «[...] l'étude de l'interaction entre humains, ordinateurs et tâches. Elle s'attache principalement à comprendre comment les humains et les ordinateurs peuvent accomplir des tâches interactivement et comment de tels systèmes interactifs sont conçus ».

D'après [KAY 89], l'EAD est l' «enseignement qui, ou bien ne nécessite pas la présence physique de l'enseignant payé pour le dispenser à l'endroit où il est reçu, ou bien dans lequel l'enseignant est présent pour des tâches ou des événements particuliers. » Ces tâches, ajoute [KAY 89], peuvent être des sessions de conseil, tutorat, séminaire ou de résolution de problèmes. Cette définition, très générale, demande à être précisée, c'est ce que fait [HOL 81, cité par KAY 89], en indiquant six caractéristiques de l'EAD :

l'utilisation de cours conçus principalement pour être étudiés ;

l'existence de communications organisées, bidirectionnelles, entre les élèves et une organisation telle qu'une université, un lycée ou une école avec ses tuteurs et conseillers ;

l'organisation planifiée et explicite de l'étude individuelle ;

l'utilisation à des fins éducatives de méthodes de communication de groupes, quand de nombreux élèves suivent le même cours, afin d'en réduire le coût ;

l'application de méthodes de production industrielles pour la production de matériels d'apprentissage et pour l'administration de pratiques d'EAD;

la notion d'étude à distance comme une forme «médiatée ${ }^{\text {» }}$ de conversation didactique guidée.

Les frontières respectives de ces différents champs sont loin d'être évidentes à établir et varient selon les auteurs. Si le HCI apparait être le plus général, le CMC peut être perçu comme une partie du CSCW ou, au contraire, l'englober. À lire les récents actes de colloques, c'est le CSCW qui semble être le plus susceptible de fonder un champ de recherche autonome.

5 Avec [BRE 96], nous préférons employer le terme d'enseignement «médiaté » pour «opéré par voie de télécommunications », plutôt que celui de " médiatisé », qui signifie « diffusé par un quelconque média ». 
Ces différents champs de recherche contribuent à produire tant de données qu'il a fallu opérer une sélection. Nous avons retenu, sans prétention à l'exhaustivité, les études :

- récentes, c'est-à-dire réalisées dans les années quatre-vingt-dix ; il n'était pas, en effet, possible de reprendre un historique complet de tous ces champs de recherche. Nous avons préféré nous centrer sur les études récentes, laissant le lecteur se reporter à des auteurs ayant édité des compilations d'articles fondateurs [GRE 88].

- inscrites dans un contexte éducatif ou dont les résultats sont transférables dans un tel contexte. Pour ce faire, les études devront être centrées sur les indices de la communication ou l'enseignement plutôt que sur le contenu, propre à une démarche de type didactique.

— où au moins un groupe de sujets est placé dans une situation :

- décrivant une communication ou un enseignement à distance, sans contact visuel direct, ce qui permet d'éliminer les études mettant en œuvre un travail se déroulant uniquement dans une même salle de réunion ;

- où le média utilisé est électronique, de manière à se centrer sur les apports des nouvelles techniques de l'information et de la communication. Ce qui élimine les études centrées uniquement sur l'enseignement par correspondance ;

- de groupe-contrôle avec une communication ou un enseignement en face à face, ce qui permet, une fois de plus, de vérifier expérimentalement les mérites supposés de ces nouvelles techniques.

- où les variables dépendantes mesurées :

- sont comparées inter- ou intragroupes, à l'aide de tests statistiques appropriés, critère qui garantit que les différences intermédias sont bien significatives ;

- ne sont pas uniquement basées sur la satisfaction des sujets, de manière à atténuer l'effet « attrait pour la nouveauté » déjà cité dans notre introduction.

Pour faciliter la lecture, la quinzaine d'études retenues a été classée en trois catégories (1) les études centrées sur la communication interpersonnelle, dont les résultats peuvent être utiles aux recherches sur l'EAD, leur but commun étant de 
favoriser la communication inter- et intragroupes ; (2) les études centrées sur une production, qu'elles soient de type coopératif ou collaboratif, ces études mettent en place des situations où des sujets résolvent un problème en groupe, en utilisant des ressources humaines et matérielles communes, ce qui n'est donc pas très éloigné d'un contexte éducatif ; (3) les études se réclamant de l'EAD et mettant en œuvre un suivi de cours, qui ont bien évidemment une place centrale ici.

Afin d'affiner encore ce classement, un deuxième critère est utilisé, le type de variable dépendante mesurée, réparti en trois catégories classiques dans la littérature sur l'enseignement [DUN 86] mais également dans celle sur l'EAD, avec d'autres dénominations, chez [COL 90] ou encore [ROM 90]: variables contextuelles, de processus et de produit. Les premières ont trait principalement aux caractéristiques des sujets et de l'environnement, les deuxièmes aux événements se produisant pendant le cours et les dernières aux résultats des élèves, en termes d'apprentissage et de réussite. Le tableau I ci-dessous rassemble toutes les études recensées selon ces catégories.

Tableau I. Vue générale des études sur l'EAD, selon le type de média utilisé

\begin{tabular}{|c|c|c|c|c|c|c|c|c|c|}
\hline $\begin{array}{l}\text { Caté- } \\
\text { gorie }\end{array}$ & Auteurs & $\begin{array}{c}\text { Voix } \\
\text { seule } \\
\text { sur } \\
\text { cas- } \\
\text { sette } \\
\end{array}$ & $\begin{array}{l}\text { Audio } \\
\text { confé- } \\
\text { rence }\end{array}$ & $\begin{array}{c}\text { Audio } \\
\text { confé- } \\
\text { rence } \\
\text { et } \\
\text { diapos } \\
\end{array}$ & CMC & $\begin{array}{l}\text { CMC } \\
\text { et } \\
\text { télé- } \\
\text { phone }\end{array}$ & $\begin{array}{l}\text { TV } \\
\text { inter- } \\
\text { acti- } \\
\text { ve }\end{array}$ & $\begin{array}{l}\text { Visio- } \\
\text { confé- } \\
\text { rence }\end{array}$ & $\begin{array}{l}\text { Centration } \\
\text { des } \\
\text { variables } \\
\text { dépendantes }\end{array}$ \\
\hline $\begin{array}{l}\text { Com- } \\
\text { muni- } \\
\text { cation }\end{array}$ & $\begin{array}{l}\text { [O'CO 93] } \\
\text { [PÉR 92a] } \\
\text { [SEL 95] }\end{array}$ & & $\sqrt{ }$ & & & & & $\begin{array}{l}\sqrt{ } \\
\sqrt{ } \\
\sqrt{ }\end{array}$ & $\begin{array}{l}\text { Processus } \\
\text { Processus } \\
\text { Processus }\end{array}$ \\
\hline $\begin{array}{l}\text { Pro- } \\
\text { duc- } \\
\text { tion }\end{array}$ & $\begin{array}{l}\text { [AND 96] } \\
\text { [GAL 90] } \\
\text { [GAL 92] } \\
\text { [LEB 96] }\end{array}$ & & $\sqrt{ }$ & & $\begin{array}{l}\sqrt{ } \\
\sqrt{ } \\
\sqrt{ }\end{array}$ & $\begin{array}{l}\sqrt{ } \\
\sqrt{ }\end{array}$ & & $\sqrt{ }$ & $\begin{array}{l}\text { Processus } \\
\text { Produit } \\
\text { Produit } \\
\text { Processus } \\
\end{array}$ \\
\hline $\begin{array}{l}\text { Suivi } \\
\text { de } \\
\text { cours }\end{array}$ & $\begin{array}{l}\text { [GEI 90] } \\
{[\text { HIL 94] }} \\
{[\text { LEM 96] }} \\
{[\text { MIL 96] }} \\
\text { [PAR 95] } \\
\text { [SEI 90] } \\
\text { [STO 90] }\end{array}$ & $\sqrt{ }$ & & $\sqrt{ }$ & $\begin{array}{l}\sqrt{ } \\
\sqrt{ }\end{array}$ & & $\begin{array}{l}\sqrt{ } \\
\sqrt{ }\end{array}$ & $\sqrt{ }$ & $\begin{array}{l}\text { Produit } \\
\text { Produit } \\
\text { Processus } \\
\text { Produit } \\
\text { Contexte } \\
\text { Produit } \\
\text { Produit } \\
\end{array}$ \\
\hline
\end{tabular}




\subsection{Les études centrées sur la communication interpersonnelle}

Les études centrées sur la communication interpersonnelle mettent toutes en scène un ou plusieurs groupes de sujets communiquant via visioconférence, en vue de converser ou de prendre une ou plusieurs décisions de travail. Elles sont majoritairement axées sur les processus, c'est-à-dire les paramètres conversationnels (tours de paroles, silences, etc.) et comportementaux, ainsi que sur la qualité du média utilisé. Le tableau II ci-dessous reprend l'essentiel des études centrées sur la communication interpersonnelle, toutes axées sur le processus.

Tableau II. Résumé des études centrées sur la communication interpersonnelle.

\begin{tabular}{|c|c|c|c|}
\hline Auteurs & Facteurs manipulés & Procédure & Résultats principaux \\
\hline [O'CO 93] & $\begin{array}{l}\text { Face à face } v s \text { deux } \\
\text { types de } \\
\text { visioconférence. }\end{array}$ & $\begin{array}{l}\text { Réunion de travail. } \\
\text { Centration sur les } \\
\text { principaux } \\
\text { paramètres de la } \\
\text { conversation. }\end{array}$ & $\begin{array}{l}\text { Dialogue à distance plus } \\
\text { formel : interruptions et } \\
\text { recouvrements plus } \\
\text { fréquents, tours de parole } \\
\text { plus longs. }\end{array}$ \\
\hline [PÉR 92a] & $\begin{array}{l}\text { Face à face } v s \\
\text { visioconférence. }\end{array}$ & $\begin{array}{l}\text { Réunion de travail } \\
\text { d'ingénieurs, } \\
\text { centration sur les } \\
\text { indices non-verbaux } \\
\text { de la conversation. }\end{array}$ & $\begin{array}{l}\text { La visioconférence prive } \\
\text { les sujets d'une } \\
\text { information sur la } \\
\text { vectorisation du regard. }\end{array}$ \\
\hline [SEL 95] & $\begin{array}{l}\text { 1. Face à face } v s \\
\text { visioconférence. } \\
2 \text {. Visioconférence } \\
v s \\
\text { audioconférence. }\end{array}$ & $\begin{array}{l}\text { Réunion de travail. } \\
\text { Deux systèmes de } \\
\text { visioconférence de } \\
\text { qualité différente } \\
\text { sont comparés. }\end{array}$ & $\begin{array}{l}\text { Le pourcentage de paroles } \\
\text { simultanées est supérieur } \\
\text { en face à face. } \\
\text { La durée entre deux tours } \\
\text { de parole est inférieure en } \\
\text { face à face. } \\
\text { Les sujets en face à face } \\
\text { ont l'impression d'un } \\
\text { meilleur contrôle de la } \\
\text { conversation. }\end{array}$ \\
\hline
\end{tabular}

Citons tout d'abord une étude récente sur la communication par vidéo réalisée par [O'CO 93] qui cherchent à identifier les modifications induites par des 
dispositifs de ce type sur la communication entre deux personnes. Différents paramètres de la conversation naturelle sont répertoriés (les acquiescements, les interruptions, les recouvrements de parole, le nombre de tours de parole, leur longueur moyenne, etc.) et étudiés au travers de trois modes de conversation : la conversation face à face et deux dispositifs de qualité différente. L'hypothèse est que les dispositifs qui produisent une qualité d'image et de son optimale, avec des temps de réponse négligeables, devraient produire des formes de conversation analogues à la conversation naturelle, alors qu'une dégradation des caractéristiques techniques (vidéo de mauvaise qualité, temps de transmission important) devrait affecter les paramètres élémentaires de la conversation. Quatorze communications ont été enregistrées (entre des groupes de quatre à neuf sujets), desquelles ont été extraits et analysés par deux juges des segments de vingt minutes. Les résultats montrent que le dispositif de bonne qualité est intermédiaire entre les deux autres, sans jamais cependant être équivalent à la conversation en face à face. Ainsi, le nombre d'acquiescements, d'interruptions et de recouvrements de parole est plus élevé dans la conversation naturelle alors que la taille des tours de parole y est moindre. Le dialogue, rendu plus formel lorsqu'un dispositif technique s'intercale entre les interlocuteurs, n'est pas sans surprises et apparaît dans d'autres circonstances (communication au talkie-walkie, par exemple). Le résultat intéressant est cependant qu'un dispositif technique qui reproduit l'image et le son des participants en temps réel, procédé qui semble identique à une communication en face à face, affecte tout de même les processus de communication élémentaires de la conversation naturelle.

[PÉR 92a] a mené une étude observant des réunions de travail en face à face ou à distance et en se centrant plus particulièrement sur leur dimension «multimodale », c'est-à-dire le non verbal. Il a observé (1) comment sont distribués les échanges de parole dans le groupe et (2) comment est organisée la dimension pragmatique de ces échanges. Concernant le premier point, il montre combien la situation à distance prive les participants de la focalisation non verbale de la parole, c'est-à-dire de tous les regards, postures en direction d'un autre participant qui les incitent à prendre un tour de parole (l'auteur parle d'adressage) et qui ne nuisent pas 
à la fluidité de la conversation comme nuiraient des interpellations nominatives. De même, les regards vers l'interlocuteur sont beaucoup plus importants en situation à distance, où il s'agit d'observer finement sa réaction («manifestations mimogestuelles et infra verbales attestant la volonté, l'impatience ou simplement la volonté de prendre la parole»id., p. 66) et éventuellement lui céder un tour de parole. L'auteur montre, de plus, que dans le cas d'audioconférences seul le contenu de l'intervention et les messages tels que raclements de gorge permettent de négocier l'alternance de la parole. Il établit, de manière très judicieuse, les deux fonctions du regard : recueillir une information sur l'interlocuteur et aussi l'informer sur une cession de la parole, ce qui est difficile à rendre par le biais d'un système à distance. Cette absence de «vectorisation du regard» est sans doute un des manques les plus patents des nouveaux systèmes de visioconférence. À propos du deuxième point, la dimension pragmatique ${ }^{6}$, [PÉR 92a] trouve beaucoup moins de différences entre les deux situations: pas de différences en ce qui concerne la structuration du discours, en revanche, de nombreuses différences en ce qui concerne la gestuelle (marques d'impatience, d'accord ou de désaccord) et les expressions faciales (sourires), ce qui va de soi. Plus intéressantes sont les caractéristiques des silences : deux fois plus nombreux en situation à distance, montrant une demande implicite de feed-back de la part du locuteur envers ses pairs.

L'expérimentation de [SEL 95] a un but semblable, l'effet des médias sur la conversation est testé dans deux expérimentations : (1) en face à face dans la même pièce vs diffusé par visioconférence (deux systèmes différents) ; (2) visioconférence (deux systèmes différents) vs par audioconférence. Les participants de chaque groupe commencent par faire connaissance, pendant quinze minutes, puis on leur présente le groupe « à distance » avec lequel ils devront débattre, pendant seize

6 Les éditeurs de l'ouvrage [PÉR 92b] paraissent adopter une définition beaucoup plus large de la pragmatique, portant d'habitude uniquement sur le discours : « [...] l'ensemble des procédures qui aident à l'accomplissement des buts poursuivis dans la rencontre » et comprenant donc des aspects non verbaux. 
minutes. Ils doivent ensuite remplir un questionnaire de type échelle de Likert sur leurs impressions à propos de la séance. Si aucune différence significative n'a été mise au jour concernant le nombre, la durée et la distribution des tours de parole, on observe des différences significatives dans les domaines suivants ${ }^{7}$ :

- le pourcentage de paroles simultanées est significativement supérieur en face à face que dans les visioconférences ;

- la durée entre deux tours de parole est significativement inférieure en face à face que dans les vidéoconférences.

À propos du questionnaire d'opinion, les sujets en face à face ont été beaucoup plus nombreux que ceux en visioconférence à penser qu'ils avaient un bon contrôle de la conversation et une meilleure écoute de leur partenaires.

Ces études délaissent quelque peu un phénomène pourtant primordial, celui du processus de prise de décision ou, plus généralement, de production : le champ de recherche que nous détaillons maintenant se préoccupe justement de mieux comprendre ce phénomène.

\subsection{Les études centrées sur la production}

Les études centrées sur la production (d'écrits, de planifications, etc.) intéressent encore plus directement que les précédentes le champ de l'EAD, en ce que les tâches demandées aux sujets peuvent être aisément converties en tâches d'apprentissage, si elles ne le sont pas déjà. Le tableau III ci-dessous reprend les principales caractéristiques de ce type d'études. Nous présentons des études centrées sur le processus, puis celles de [GAL 90 ; GAL 92], de type évaluatif, centrées sur le produit.

7 Nous ne présentons pas les nombreux résultats concernant les paroles simultanées ou le type de prise de tour de parole. 
Tableau III. Résumé des études centrées sur la production.

\begin{tabular}{|c|c|c|c|}
\hline Auteurs & Facteurs manipulés & Procédure & Résultats principaux \\
\hline [AND 96] & $\begin{array}{l}\text { Face à face } v s \text { voix } \\
\text { seule et voix seule } \\
v s \text { visioconférence. }\end{array}$ & $\begin{array}{l}\text { Planification d'un } \\
\text { itinéraire de voyage } \\
\text { avec des contraintes. } \\
\text { On mesure les } \\
\text { échanges et le respect } \\
\text { des contraintes. }\end{array}$ & $\begin{array}{l}\text { Les échanges sont plus } \\
\text { brefs et les changements } \\
\text { de décisions plus } \\
\text { importants en face à face. } \\
\text { Aucune autre différence } \\
\text { significative observée, } \\
\text { notamment sur la } \\
\text { performance des sujets. }\end{array}$ \\
\hline [GAL 90] & $\begin{array}{l}\text { Face à face } v s \text { CMC } \\
\text { et téléphone } v s \\
\text { CMC seul. }\end{array}$ & $\begin{array}{l}\text { Projet d'écriture de } \\
\text { documents par } \\
\text { groupes de trois. On } \\
\text { reporte le type } \\
\text { d'activité, le } \\
\text { jugement des sujets et } \\
\text { la qualité de la } \\
\text { production. }\end{array}$ & $\begin{array}{l}\text { Le média n'affecte pas la } \\
\text { qualité de la production, } \\
\text { il affecte le jugement des } \\
\text { sujets quant à la difficulté } \\
\text { de la tâche (elle semble } \\
\text { plus facile en face à face). } \\
\text { L'ajout du téléphone au } \\
\text { CMC est peu sensible. }\end{array}$ \\
\hline [GAL 92] & $\begin{array}{l}\text { Face à face } v s \text { CMC } \\
v s \ll \text { libre choix } .\end{array}$ & $\begin{array}{l}\text { Projet d'écriture de } \\
\text { documents par } \\
\text { groupes de trois. On } \\
\text { reporte le type } \\
\text { d'activité, le } \\
\text { jugement des sujets et } \\
\text { la qualité de la } \\
\text { production. }\end{array}$ & $\begin{array}{l}\text { Les sujets en libre choix } \\
\text { préfèrent être en face à } \\
\text { face lorsque le projet est } \\
\text { de type analyse-synthèse } \\
\text { de document. Ces mêmes } \\
\text { sujets préfèrent rester en } \\
\text { face à face, même lorsque } \\
\text { les exigences de la } \\
\text { communication sont plus } \\
\text { faibles. La performance } \\
\text { des groupes est } \\
\text { indépendante du média. }\end{array}$ \\
\hline [LEB 96] & $\begin{array}{l}\text { Face à face } v s \\
\text { ordinateur (CMC). }\end{array}$ & $\begin{array}{l}\text { Écriture d'un rapport. } \\
\text { Mesure du nombre de } \\
\text { messages. }\end{array}$ & $\begin{array}{l}\text { Le nombre de messages } \\
\text { est plus faible en CMC } \\
\text { qu'en face à face. }\end{array}$ \\
\hline
\end{tabular}


[AND 96] mettent en place une situation assez proche de celle de [CHA 88] ${ }^{8}$, afin d'étudier les processus de résolution de problèmes en groupe, à travers une tâche de planification d'itinéraire, dans le cadre d'une simulation d'une agence de voyage. Nous décrivons ici la première des trois études réalisées par les auteurs, car elle seule correspond aux critères fixés supra au § 3.1. Dans cette étude, donc, les sujets $(\mathrm{N}=40)$ doivent communiquer un itinéraire à un « agent de voyage », faisant partie de l'équipe de recherche, itinéraire devant répondre à des contraintes financières, de durée et de lieu. Deux comparaisons sont faites : un travail sur papier, en face à face, est comparé à une liaison audio seulement, où les protagonistes sont dans deux pièces séparées ; cette même configuration étant aussi comparée à un travail sur ordinateur, de type visioconférence, où les sujets peuvent visualiser le visage de leur interlocuteur ainsi que la carte de l'itinéraire. Les résultats montrent que, quelles que soient les conditions, il n'y a aucune différence significative dans la performance, mesurée en nombre de villes visitées. En ce qui concerne les caractéristiques de l'échange, on peut seulement mettre au jour une longueur des échanges moindre en face à face, comparé à l'audio seul. Le processus de décision des sujets comprend également significativement plus de changements volontaires en situation de face à face qu'en audio seul. Toutes les autres comparaisons ne sont pas significatives. Pour résumer, il ne semble pas que la moindre présence sociale, dans les conditions autres qu'en face à face, nuise à la qualité du processus de résolution de problème de type planification de trajet.

\footnotetext{
8 La célèbre étude de [CHA 88], que nous ne recensons pas ici du fait de son ancienneté, a permis d'évaluer l'effet du canal sur la qualité des communications. L'auteur a mesuré les durées moyennes de résolution de problèmes (dans une tâche de localisation sur carte) mis par deux personnes, selon qu'elles se communiquent librement en face à face ou bien dans les modalités suivantes : (1) voix seulement, (2) par l'écrit à la main, (3) par l'écrit sur machine à écrire. Les principaux résultats montrent que les canaux où la durée de résolution de problèmes est la plus courte sont les canaux vocaux. De plus, ces canaux sont également les plus verbeux. Les canaux passant par l'écrit sont, eux, les moins redondants.
} 
[LEB 96] comparent une tâche de rédaction collaborative de texte, en face à face et à distance. À six reprises, une trentaine de groupes d'environ quatre étudiants doivent rédiger un rapport, soit autour d'une table, soit à l'aide d'un logiciel de communication qui leur permet de s'échanger des messages et de visualiser l'avancement du texte. Cette recherche vise notamment à vérifier le principe de «blocage de la production », défini en psychologie sociale par [DIE 91], selon lesquels la communication en face à face souffrirait de la séquentialité de la production des messages, alors que la communication assistée par ordinateur favoriserait un plus grand nombre de messages, donc d'idées, grâce à leur production simultanée. Les résultats montrent que ce principe n'est pas suivi : la difficulté de la communication par ordinateur conduit à un nombre d'actes de communication par minute 2,6 fois plus faible qu'en face à face, tout comme le suggèrent d'autres études similaires. Au stade actuel de la technologie, la communication textuelle d'un groupe à distance ne permet donc pas d'atteindre les performances, en termes d'informations par minute, de la communication face à face.

Passons aux deux études de type évaluatif. Dans la première étude de [GAL 90], les sujets $(\mathrm{N}=117)$ doivent mettre en œuvre un projet d'écriture en groupe, sous forme d'étude de cas, en utilisant un environnement de type CMC. Pour ce faire, des groupes de trois étudiants sont aléatoirement constitués, chaque étudiant participant à deux travaux différents de deux semaines chacun. Deux facteurs sont manipulés : (1) la segmentation du projet (project divisibility), avec deux modalités : • projet segmenté, où les étudiants doivent rédiger différentes lettres de licenciement, ou - projet intégré, où les étudiants doivent analyser les problèmes de personnel d'une entreprise et rédiger un document de synthèse ; (2) la modalité de communication : - ordinateur seul, où toutes les communications dans le groupe se passent via un système $\mathrm{CMC}$, ordinateur et téléphone, et • en face à face, dans une même salle de réunion. Les résultats montrent que les étudiants en face à face planifient significativement plus tôt leur projet que dans les deux autres modalités et qu'ils cessent de planifier pour se consacrer aux autres phases de rédaction également plus tôt. Les étudiants dans la modalité « ordinateur seul » passent significativement plus 
de temps à communiquer que dans les deux autres modalités. En outre, aucune différence significative n'est mesurée selon le type d'écrit fourni. Un questionnaire passé aux sujets montre qu'ils ont l'impression de rencontrer beaucoup plus de problèmes de coordination, et de réaliser un travail de moindre qualité dans les modalités avec ordinateur qu'en face à face. Curieusement, cette impression n'est pas confirmée par la qualité du travail, lorsqu'elle est évaluée par des juges : tous les travaux sont jugés de qualité égale. Cette étude montre que si la communication via $\mathrm{CMC}^{9}$ est perçue comme plus frustrante et moins efficace par les opérateurs, elle ne s'accompagne pas d'une moins bonne qualité des productions écrites.

Les mêmes auteurs [GAL 92] ont répliqué cette étude avec les mêmes modalités et des contenus et une procédure sensiblement différents. La procédure comprenait, à la place du groupe CMC seul, un groupe « libre choix » qui était libre de décider de leur média de communication. Les auteurs tentent ainsi de valider la théorie de la contingence, qui prédit que des sujets, face à une tâche de communication, choisissent le média le plus adapté à cette tâche. Placés face à une tâche de résolution de problème faiblement structuré, les sujets devraient choisir un média le plus « riche » possible (le face à face) et vice versa. Les résultats montrent que cette hypothèse n'est que partiellement vérifiée. Les étudiants en libre choix utilisent significativement plus le face à face lorsque le projet est de type intégré (faire une analyse puis une synthèse) et que la tâche est une tâche de planification. En revanche, deux incohérences avec la théorie sont relevées : (1) le groupe «libre choix », lors des phases requérant moins de communication, ne préfère pas un média moins riche, et continue de communiquer en face à face ; (2) une fois de plus, la performance des groupes ne dépend pas du média utilisé. Les auteurs invoquent la capacité d'adaptation du comportement humain pour effectuer une tâche complexe, quel que soit l'environnement considéré.

\footnotetext{
9 L'ajout d'un téléphone au système CMC n'améliore guère l'opinion des sujets.
} 
Passons enfin à ce qui constitue notre propos central, les études de suivi de cours à distance.

\subsection{Les études centrées sur le suivi de cours}

Nous terminons cette revue de question par les études mettant en place une situation de suivi de cours, en EAD. Le tableau IV ci-dessous en reprend les principales caractéristiques. Plutôt que de décrire les études selon la centration de leurs variables dépendantes, nous préférons tout d'abord mentionner celles mettant au jour des différences intermédias. 
Tableau IV. Résumé des études centrées sur le suivi de cours.

\begin{tabular}{|c|c|c|c|}
\hline Auteurs & Facteurs manipulés & Procédure & Résultats principaux \\
\hline [GEI 90] & $\begin{array}{l}\text { Cours traditionnel } \\
\text { vs téléconférence vs } \\
\text { télécours. }\end{array}$ & $\begin{array}{l}\text { Résultats aux cours de } \\
\text { gestion de ressources } \\
\text { humaines. }\end{array}$ & $\begin{array}{l}\text { Les techniques « à } \\
\text { distance » donnent de } \\
\text { meilleurs résultats que le } \\
\text { cours traditionnel. }\end{array}$ \\
\hline [HIL 94] & $\begin{array}{l}\text { Cours par } \\
\text { ordinateur vs cours } \\
\text { traditionnel } v s \\
\text { mixage des deux. }\end{array}$ & $\begin{array}{l}\text { Cours universitaires } \\
\text { sur diverses matières. } \\
\text { On mesure la réussite } \\
\text { des étudiants aux } \\
\text { examens. }\end{array}$ & $\begin{array}{l}\text { Aucune différence } \\
\text { significative intergroupes, } \\
\text { hormis le cours } \\
\text { d'informatique. }\end{array}$ \\
\hline [LEM 96] & $\begin{array}{l}\text { Cours en face à face } \\
\text { vs audioconférence } \\
\text { avec transparents. }\end{array}$ & $\begin{array}{l}\text { Cours de sciences } \\
\text { économiques. } \\
\text { Centration sur les } \\
\text { aspects } \\
\text { morphosyntaxiques du } \\
\text { discours de } \\
\text { l'enseignant. }\end{array}$ & $\begin{array}{l}\text { Discours à distance plus } \\
\text { complexe qu'en } \\
\text { présence : débit plus } \\
\text { élevé, phrases moins } \\
\text { redondantes, plus } \\
\text { d'organisateurs } \\
\text { argumentatifs. }\end{array}$ \\
\hline [MIL 93] & $\begin{array}{l}\text { Cours en présence } \\
\text { vs vidéo interactive, } \\
\text { donné } \\
\text { successivement à } \\
\text { deux groupes, } \\
\text { étudiant en } \\
\text { présence ou à } \\
\text { distance. }\end{array}$ & $\begin{array}{l}\text { Cours de sciences de } \\
\text { l'éducation. On } \\
\text { mesure l'attitude des } \\
\text { étudiants à propos de } \\
\text { leur apprentissage et } \\
\text { de leur communication } \\
\text { pendant } \\
\text { l'enseignement. }\end{array}$ & $\begin{array}{l}\text { Différence significative } \\
\text { sur l'attitude : les } \\
\text { étudiants à distance ont } \\
\text { une meilleure opinion de } \\
\text { l'EAD que ceux en } \\
\text { présence. Effet sur } \\
\text { l'apprentissage effectif } \\
\text { supérieur en présence. }\end{array}$ \\
\hline [PAR 95] & $\begin{array}{l}\text { EAD (cassette audio } \\
\text { ou conférence } \\
\text { informatisée ou } \\
\text { correspondance) vs } \\
\text { en présence. }\end{array}$ & $\begin{array}{l}\text { Cours d'anglais et de } \\
\text { sociologie. Taux } \\
\text { d'achèvement des } \\
\text { études en fonction des } \\
\text { caractéristiques } \\
\text { personnelles des } \\
\text { étudiants. }\end{array}$ & $\begin{array}{l}\text { Le locus de contrôle et les } \\
\text { finances des étudiants } \\
\text { sont liés avec l'abandon } \\
\text { des études. }\end{array}$ \\
\hline [SEI 90] & $\begin{array}{l}\text { Cours par télévision } \\
\text { interactive } v s \text { en } \\
\text { présence. }\end{array}$ & $\begin{array}{l}\text { Réussite des étudiants- } \\
\text { ingénieurs aux } \\
\text { examens. }\end{array}$ & $\begin{array}{l}\text { Aucune différence } \\
\text { significative entre les } \\
\text { deux types de cours. }\end{array}$ \\
\hline [STO 90] & $\begin{array}{l}\text { Cours traditionnel } \\
v s \text { cours vidéo vs } \\
\text { cours vidéo } \\
\text { interactif. }\end{array}$ & $\begin{array}{l}\text { On observe la réussite } \\
\text { à une année d'études } \\
\text { d'étudiants-ingénieurs. }\end{array}$ & $\begin{array}{l}\text { Aucune différence } \\
\text { significative intergroupes. }\end{array}$ \\
\hline
\end{tabular}


[GEI 90] mesurent la réussite des étudiants à deux types de cours à distance en gestion des ressources humaines, assistés par ordinateur : - téléconférence (virtual lab, mêlant des téléconférences à heure précise et des sessions en face à face) ; - télécours (cours sur bandes vidéo et correspondance par courrier électronique) et la comparent à celle obtenue dans un cours «traditionnel ». Les résultats montrent que la meilleure réussite est obtenue avec le télécours et avec la téléconférence, tous deux étant significativement supérieurs à l'enseignement traditionnel. Les auteurs, toutefois, signalent que les étudiants des cours à distance ont un emploi, sont plus âgés et par conséquent plus motivés par la poursuite de leurs études.

[LEM 96] mettent en évidence, en utilisant entre autres la méthode définie par Bronckart (voir § 2.2.), les différences entre un discours d'enseignant produit en amphithéâtre et un discours produit à distance à l'aide d'un dispositif audiographique. En raison vraisemblablement de l'importante activité de préparation des supports visuels de cours ainsi que de la suppression des informations d'ordre analogique en provenance des étudiants (froncements de sourcils, regards sur le voisin, etc.), le discours produit à distance se révèle plus complexe qu'en situation traditionnelle : les phrases y sont syntaxiquement plus justes, moins redondantes, le nombre d'anaphores et d'organisateurs argumentatifs y est plus important, le débit y est plus élevé. L'enseignant avait anticipé sur ce résultat en diminuant la durée des séquences de cours pour les étudiants à distance, pressentant une charge cognitive plus importante.

L'étude de [MIL 93] a pour objet de répondre à la question suivante : « Est-ce que les étudiants diffèrent, selon qu'ils apprennent "en direct" ou "à distance", dans leur impression de maîtriser le contenu du cours, de pouvoir participer aux discussions de groupe ainsi que dans leur réelle maîtrise du contenu » (p. 53). Cette étude est donc évaluative, en combinant des éléments subjectifs - les deux premiers, les attitudes - et objectifs - le dernier, la maitrise du contenu. L'expérimentation porte sur deux cours de maitrise en sciences de l'éducation (psychologie de la lecture et recherche expérimentale), sur six séances de deux heures. Les étudiants proviennent de deux structures d'enseignement, en présence 
(on campus, $\mathrm{N}=22$ ) ou à distance (off campus, $\mathrm{N}=29$ ), ils suivent alernativement deux types de présentation, l'une en direct, l'autre à distance, par un système vidéo interactif bidirectionnel. Chaque groupe d'étudiants suit donc, dans le cadre de l'expérimentation, trois cours « en présence » et trois cours « à distance ». Ils passent ensuite un questionnaire d'attitude sur les deux variables sus-mentionnées, ainsi qu'un questionnaire de compréhension. Il est intéressant de noter que, à propos des attitudes (sur l'apprentissage et l'interaction), seuls les étudiants inscrits à distance (off campus) ne perçoivent pas de différence entre cours à distance et en présence. En revanche, les étudiants on campus préfèrent significativement les cours en direct. Concernant l'apprentissage effectif, mesuré par un questionnaire à choix multiple, on trouve une différence significative globale selon que le cours est en présence (92\% de bonnes réponses) ou à distance (86\% de bonnes réponses). On trouve également un effet-contenu, avec un cours mieux compris que l'autre. Ces résultats sont cohérents avec ceux de [SAL 84], montrant qu'il y a un lien entre l'attitude des élèves à propos d'un média et leur réussite effective dans l'apprentissage. Les étudiants réussiraient moins bien à distance s'ils pensent qu'il est plus facile d'étudier avec un média télévisuel et donc investissent moins « d'effort mental » (amount of invested mental effort) dans l'apprentissage.

[PAR 95] s'emploie à déterminer quels facteurs peuvent prédire l'achèvement d'études à distance. Les facteurs en question sont de type contextuel ${ }^{10}$ et essentiellement centrés sur les caractéristiques des étudiants : locus de contrôle, âge, sexe, nombre de cours à distance réussis, moyens financiers et nombre d'heures d'étude. Les cours sont donnés en présence $(\mathrm{N}=94)$ ou avec trois médias différents, à distance $(\mathrm{N}=76)$. Les résultats montrent que seuls le locus de contrôle et l'assistance financière sont liés avec l'abandon des études et prédisent même jusqu'à $85 \%$ des abandons en cours à distance. Ce résultat a été complété par des

\footnotetext{
10 Cette étude est la seule que nous ayons pu trouver qui soit centrée sur ces caractéristiques contextuelles.
} 
interviews d'étudiants justifiant également leur abandon par des arguments « externes » : emploi, famille, manque d'équipement informatique.

Afin d'être complet, il nous faut citer également des études dont les résultats n'ont pas montré de différences significatives en faveur de l'EAD. Il s'avère que ce type d'études a moins de chances de se trouver publié, les normes de production scientifique s'intéressant plutôt à des différences significatives, ce que [CLA 83] nomme des distorsions d'effets dus à des décisions éditoriales.

[HIL 94], l'une des pionnières de la communication par ordinateur, présente l'évaluation d'un système d'EAD, téléconférence par ordinateur (computer mediated conference) en comparant les résultats des étudiants d'un tel groupe $(\mathrm{N}=148)$ avec un groupe-contrôle $(\mathrm{N}=89)$ et un groupe utilisant une combinaison des deux précédents $(\mathrm{N}=96)$. Les cours portent sur l'informatique, les mathématiques, le management, la sociologie et les statistiques. Que le cours soit en présence ou à distance, aucune différence significative n'est mise au jour dans les performances des étudiants, que ce soit à l'examen partiel ou final. La seule différence trouvée est à propos du cours d'informatique, on peut penser que l'utilisation d'un ordinateur pour communiquer a pu interférer avec le contenu du cours. Précisons de plus que nous extrayons du travail de [HIL 94] une très faible partie, l'auteur ayant utilisé bien d'autres moyens d'évaluer le travail d'étudiants dans ce qu'elle nomme une « classe virtuelle » (virtual classroom).

[SEI 90] comparent la réussite aux examens d'étudiants-ingénieurs à distance, via télévision interactive - bandes vidéo et retour audio pour les questionsréponses $(\mathrm{N}=69)$ et en présence $(\mathrm{N}=99)$. Les cours portent sur l'informatique et l'électricité. Les résultats ne montrent aucune différence significative entre les cours en présence et à distance.

[STO 90] mène une recherche faisant intervenir 8341 étudiants-ingénieurs, certains à distance, avec un cours sur bandes vidéo, comprenant ou non une possibilité de questions (interactif); d'autres en présence, dans un cours traditionnel. Les résultats, en mesurant la réussite à une année d'études montrent qu'aucune différence significative entre les groupes n’est trouvée, même si les groupes à distance avec bande vidéo non interactive ont des pourcentages de 
réussite un peu meilleurs que les autres. L'auteur conclut à l'intérêt de tels cours à distance, puisqu'ils permettent d'obtenir des résultats du même ordre que s'ils étaient menés en présence.

Les résultats de cette partie, en majorité non significatifs, nous confirment dans le commentaire de [KOZ 91] : si les effets d'un tel média sur l'apprentissage sont si faibles, c'est aux chercheurs de faire en sorte qu'à l'avenir un média puisse être significativement efficace, comparé à un enseignement « en présence ».

\section{5. Éléments pour des recherches futures}

Après ce tour d'horizon de travaux sur l'EAD, faisons quelques commentaires prospectifs sur le traitement des variables contextuelles, de processus et de produit dans la recherche expérimentale.

À notre connaissance, il y a peu d'études qui reprennent les notions de la planification de séquences d'enseignement et les appliquent au contexte de l'EAD. Il semble, à lire certains [SAU 94 ; SCH 93 ; TAY 95], qu'il n'y aurait pas de différences notables si on le compare à l'enseignement « en présence », sur le thème duquel beaucoup a été écrit. Plus généralement, les travaux faisant intervenir le contexte comme facteur sont trop rares. Pourtant, [LEM 96] montrent que les différences observées dans le discours de l'enseignant, selon qu'il soit « en présence» ou à « distance » peut avoir sa source dans une différence de planification du cours.

Nous n'avons trouvé que très peu d'études centrées sur le processus dans une situation d'EAD ${ }^{11}$. Pourtant il en existe de nombreuses modélisant la phase

11 C'est également l'avis de [CLY 83, cités par MAR 96] : « Malgré l'accroissement des cours en EAD, il existe peu de connaissance systématique de la manière dont les étudiants utilisent les matériels d'apprentissage qui leur sont délivrés. » 
interactive de l'enseignement en présence - voir notamment [MOR 78 ; SHA 81]

et il serait intéressant de considérer leur application dans une situation d'EAD.

Concernant le « produit », on trouve, dans la littérature [JOH 91], des invites à ne pas prendre en compte uniquement l'apprentissage des élèves en EAD, mais aussi des composantes sociales de motivation, communication, etc., il nous semble que les recherches expérimentales se désinvestissent de cet aspect, qui reste, il faut l'avouer, le seul objet des études de type qualitatif. Il y a là un champ d'investigation qui nous paraît prometteur, d'autant que la psychologie sociale cognitive a élaboré des théories qui nous paraissent transposables à l'EAD.

Nous pouvons enfin nous interroger sur le rôle particulier que peut jouer la variable «média » dans l'apprentissage et/ou la communication dans un groupe d'apprentissage. À leur lecture, on peut se rendre compte combien les résultats ne sont encore guère tranchés. Les méta-analyses de [WAL 86] montrent que les effets les plus nets sur l'apprentissage des élèves ne sont pas dus, et de loin, à des «variables médias » : sur les sept variables qui augmentent la réussite des élèves de plus d'un sigma, six sont dues à l'enseignant proprement dit.

\section{Discussion}

Au terme de notre revue, autant théorique que pratique des quelques courants de l'EAD, deux questions cruciales restent posées - avec quels modèles théoriques rendre compte de l'utilisation d'EAD ? - en quoi l'EAD favorise l'apprentissage des élèves l'utilisant ? Nous avons vu, à propos de la première question, combien le flou du domaine de référence de l'EAD ne favorise pas l'ancrage de modèles robustes, surtout depuis que le clivage entre communication interindividuelle et communication de masse tend à disparaître avec l'avènement notamment des techniques de visioconférence [LAZ 93]. En ce qui concerne la deuxième question, replacer l'EAD en tant que média, au même titre que le livre, la télévision ou le micro-ordinateur risque d'être insuffisant. En effet, l'EAD englobe un contexte bien plus large que ses concurrents. Si l'on peut utiliser un livre ou un micro-ordinateur 
dans un nombre de contextes très diversifié (même dans celui de l'EAD, d'ailleurs), le fait de travailler en EAD oblige de facto à déterminer des éléments du contexte jamais définis dans les autres médias : l'admission des élèves (c'est le cas de l'open university britannique, par définition ouverte à tout public), le mode de transmission et d'évaluation, etc.

La distinction de [SAL 77] pour classer les différentes études sur les médias nous semble très féconde, quoique encore peu opérationnelle (voir $\S 2.4$ ). Ces auteurs distinguent les recherches expérimentales sur les médias, où ces derniers occupent une place centrale dans le dispositif ; des recherches avec les médias, où ils sont délaissés au profit d'autres préoccupations, comme la pratique de l'enseignement, la motivation ou l'apprentissage des élèves, etc. Cela rejoint la distinction que fait [COL 90] entre «évaluation » et « recherche » : la première est conçue pour déterminer la valeur d'un produit, alors que la deuxième est plutôt centrée sur la formulation d'hypothèses et la généralisabilité de ses résultats. Nous avons pu voir combien les recherches sur l'EAD s'écartaient d'études sur les médias : groupes de sujets non aléatorisés, absence fréquente de groupe contrôle, etc. Pourtant, tout indique que les chercheurs gagneraient à s'entourer de ces quelques précautions expérimentales.

En 1968, [LIC 68, cités par BOU 95], deux pionniers de la « réalité virtuelle » faisaient la prédiction suivante, qu'ils auraient pu transposer à l'enseignement : «Dans quelques années, des personnes pourront communiquer plus efficacement par machine interposée qu'en face à face ». Cette prédiction, nous l'avons vu, n'est pas largement vérifiée par les études expérimentales. Sans nous décourager, il nous semble intéressant de continuer à naviguer, selon la judicieuse formule de [SCH 96], entre rigueur et pertinence. En effet, les chercheurs en EAD sont, peutêtre plus que ceux d'autres domaines, face au dilemme suivant : mettre en place des situations très contrôlées, au risque de les rendre non pertinentes; ou bien travailler avec des situations écologiques, avec le risque qu'elles soient peu rigoureuses. 


\section{Remerciements}

Nous remercions Pascal Marquet pour ses vigilantes remarques sur une version précédente de ce travail et le Laboratoire Trigone, CUEEP, Université des Sciences et Technologies de Lille I, de nous avoir aimablement permis d'accéder à leur très riche fonds documentaire sur l'enseignement à distance.

\section{Références bibliographiques}

[AND 96] ANDERSON A. H., NEWLANDS A., MULLIN J., FLEMING A. M., DOHERTY-SNEDDON G. \& VAN DER VELDEN J., « Impact of video-mediated communication on simulated service encounters », Interaction with Computers, $8, n^{\circ} 2$, 1996, p. 193-206.

[BOU 95] BOUDOURIDES M. A., « Social and psychological effects in Computer-mediated communication », Communication au Second Workshop Neties '95, 1995, Document électronique.

[BRE 96] BRETON T. \& MAROT J.-C., " Télécommunications, téléactivités », Encyclopadia universalis, $n^{\circ}$ Suppl. 2, 1996, p. 1483-1486.

[BRO 85] BRONCKART J.-P., Le fonctionnement des discours, Delachaux et Niestlé, 1985.

[CAR 96] CARTER V., « Do media influence learning ? Revisiting the debate in the context of distance education », Open learning, 11, $n^{\circ} 1,1996$, p. 31-40.

[CAZ 86] CAZDEN C. B., « Classroom discourse », in M. C. WITTROCK (Ed.), Handbook of research on teaching, McMillan, 1986, p. 432-463.

[CHA 88] CHAPANIS A., «Interactive human communication », in I. GREIF (Ed.), Computer-supported cooperative work : a book of readings, Morgan Kaufman, 1988, p. 127-142.

[CLA 83] CLARK R. E., « Reconsidering research on learning from media », Review of Educational Research, 53, $n^{\circ} 4,1983$, p. 445-459.

[CLA 94] CLARK R. E., « Media will never influence learning », Educational Technology Research and Development, 42, $n^{\circ} 2,1994$, p. 21-29.

[CLY 83] CLYDE A. \& CROWTHER H., « How students use distance teaching materials : an institutional study », Distance Education, $n^{\circ} 4,1983$, p. 4-26.

[COL 90] COLDEWAY D. O., « Methodological issues in distance educational research », in M. G. MOORE (Ed.), Contemporary issues in american distance education, Pergamon Press, 1990, p. 386-396.

[CON 96] CONNOLLY J. H. \& PEMBERTON L., Linguistic concepts and methods in CSCW, Springer, 1996. 
[D'AM 94] D'AMBRA J. \& RICE A. E., « Multimethod approaches for the study of computer-mediated communication, equivocality and media selection », IEEE transactions on professional communication, 37, $n^{\circ} 4,1994$, p. 231-239.

[DEM 95] DE MONTMOLLIN M.,Vocabulaire de l'ergonomie, Octarès, 1995.

[DEW 94] DE WOLF H. C., « Distance education », in T. HUSÉN \& T. N. POSTLETHWAITE (Eds.), The international encyclopedia of education, Pergamon, 1994, p. 1557-1563.

[DIE 91] DIEHL M. \& STROEBE W., « Productivity loss in brainstorming groups : tracking down the blocking effect », Journal of Personality and Social Psychology, $n^{\circ} 61,1991$, p. 392-403.

[DIL s.d.] DILLENBOURG P., BAKER M., BLAYE A. \& O'MALLEY C., The evolution of research on collaborative learning, Rapport de recherche TEFCA, s. d., Document électronique.

[DIX 94] DIX A., " Computer supported cooperative work : a framework », in D. ROSENBERG \& C. HUTCHINSON (Eds.), Design issues in CSCW, Springer-Verlag, 1994, p. 9-26.

[DUN 86] DUNKIN M. J., " Concepts et modèles dans l'analyse des processus d'enseignement », in M. CRAHAY \& D. LAFONTAINE (Eds.), L'art et la science de l'enseignement, Labor, 1986, p. 39-80.

[FUL 93] FULFORD C. \& ZHANG S., « Predicting Students Satisfaction from Perception of Interaction in Distance Learning », in G. DAVIES \& B. SAMWAYS (Eds.), Proceedings of the Third Teleteaching Conference, Elsevier Science Publishers, 1993, p. 259-268.

[GAL 90] GALEGHER J. \& KRAUT R. E., « Computer-mediated communication for intellectual teamwork : a field experiment in group writing », in J. TURNER \& R. E. KRAUT (Eds.), Proc. CSCW'90, A.C.M., 1990, p. 65-78.

[GAL 92] GALEGHER J. \& KRAUT R. E., « Computer-mediated communication and collaborative writing : media influence and adaptation to communication constraints », in J. TURNER \& R. E. KRAUT (Eds.), Proc. CSCW'92, A.C.M., 1992, p. 155-162.

[GEI 90] GEITHMAN D. T. \& SLOWINSKI E., « Measuring the effectiveness of some new technologies in university education », in N. ESTES, J. HEENE \& D. LECLERCQ (Eds.), Proceedings of the 7th International conference on Technology and Education, Vol. 1, 1990, p. 159-161.

[GLI 91] GLIKMAN V. \& BARON G.-L., « Médias, multis-médias, technologies et formation à distance », Perspectives Documentaires en Education, $n^{\circ} 24,1991$, p. 63 93.

[GRE 88] GREIF I.,Computer-supported cooperative work : a book of readings, Morgan Kaufmann, 1988.

[HEN 89] HENRI F., « Distance learning and computer-mediated communication : interactive, quasi-interactive or monologue ? », in C. O'MALLEY (Ed.), Computer supported collaborative learning, Springer Verlag, 1989, p. 145-161. 
[HEN 91] HENRI F., « Computer conferencing and content analysis », in A. R. KAYE (Ed.), Collaborative learning through computer conferencing, Springer Verlag, 1991, p. 117136.

[HEN 96] HENRI F. \& RICCIARDI RIGAULT C., « Collaborative distance learning and computer conferencing », in T. T. LIAO (Ed.), Advanced educational technology : research issues and future potential, Springer Verlag, 1996, p. 45-76.

[HIL 94] HILTZ S. R., The virtual classroom, Ablex, 1994.

[HOL 81] HOLMBERG B., Status and trends of distance education, Kogan Page, 1981.

[JAC 93] JACQUINOT G., « Apprivoiser la distance et supprimer l'absence ? ou les défis de la formation à distance », Revue française de pédagogie, $n^{\circ} 102,1993$, p. 55-67.

[JOH 91] JOHNSTONE S. M., « Research on telecomunicated learning : past, present, and future », The Annals of the American Academy of Political and Social Science, $n^{\circ} 514$, 1991, p. 49-57.

[JOH 92] JOHNSON P., Human computer interaction, Mc Graw-Hill, 1992.

[JON 94] JONASSEN D., DAVIDSON M., COLLINS M., CAMPBELL J. \& HAAG B. B., «Constructivism and computer-mediated communication in distance education », The American Journal of Distance Education, 9, $n^{\circ} 2$, 1994, p. 7-26.

[KAY 89] KAYE A. R., « Distance learning systems », in M. ERAUT (Ed.), The International encyclopedia of Education technology, Pergamon Press, 1989, p. 286291.

[KIE 96] KIES J. K.,Controlled laboratory experimentation and field study evaluation of video conferencing for distance learning applications, Virginia Polytechnic Institute, 1996, rapport de recherche.

[KOZ 91] KOZMA R. B., « Learning with media », Review of educational research, 61, $n^{\circ} 2$, 1991, p. 179-211.

[KOZ 94] KOZMA R. B., "Will media influence learning ? Reframing the debate », Educational Technology Research and Development, 42, $n^{\circ} 2,1994$, p. 7-19.

[LAU 93] LAURILLARD D., Rethinking university teaching, Routledge, 1993.

[LAZ 93] LAZAR J., La science de la communication, P.U.F., 1993.

[LEB 96] LEBIE L., RHOADES J. A. \& McGRATH J. E., « Interaction process in Computer-mediated and face-to-face groups », Computer Supported Cooperative Groups, $n^{\circ}$ 4, 1996, p. 127-152.

[LEG 93] LEGENDRE R., Dictionnaire actuel de l'éducation, Guérin/Eska, 1993.

[LEM 96] LEMAIRE B., MARQUET P. \& BAILLÉ J., « Comparative analysis of teacher's discourse and students' behaviour in traditional and distance lectures », in P. CARLSON \& F. MAKEDON (Eds.), Proceedings of the World Conference on Educational Telecommunications (ED-TELECOM 96), AACE, 1996, p. 167-172.

[LIC 68] LICKLIDER J. C. R. \& TAYLOR R., " The computer as a communication device », Science and Technology, $n^{\circ} 76,1968$, p. 21-31. 
[MAR 96] MARTENS R., VALCKE M., POELMANS P. \& DAAL M., « Functions, use and effects of embedded support devices in printed distance learning materials », Learning and Instruction, 6, $n^{\circ} 1,1996$, p. 77-93.

[MAY 88] MAYER R. A. M., « Sign-manipulation resulting from new ecological conditions produced by the media », in F. LOVIS (Ed.), Remote education and informatics : teleteaching, Elsevier, 1988, p. 37-41.

[MIL 93] MILLER J. W., McKENNA M. C. \& RAMSEY P., « An evaluation of student content learning and affective perceptions of a two-way interactive video learning experience », Educational Technology, 33, $n^{\circ}$ 6, 1993, p. 51-55.

[MOO 93] MOORE M. G., " Theory of transactional distance », in D. KEEGAN (Ed.), Theoretical principles of distance education, Routledge, 1993, p. 22-38.

[MOR 78] MORINE-DERSHIMER G., « Planning in classroom reality an in-depth look », Educational Research Quarterly, 3, $n^{\circ}$ 4, 1978, p. 83-99.

[O'CO 93] O'CONAILL B. \& WHITTAKER S., « Conversations over video conferences : an evaluation of the spoken aspects of video-mediated communication », HumanComputer interaction, $n^{\circ} 8,1993$, p. 289-428.

[PAR 95] PARKER A., « Predicting dropout from distance education », in D. STEWART (Ed.), One world many voices, The Open University, 1995, p. 176-179.

[PÉR 92a] PÉRIN P., « Communication multimodale et médiatisation », in P. PÉRIN \& M. GENSOLEN (Eds.), La communication plurielle, l'interaction dans les télécommunications, La documentation française, 1992a, p. 61-83.

[PÉR 92b] PÉRIN P. \& GENSOLEN M., La communication plurielle, l'interaction dans les télécommunications, La documentation française, 1992b.

[ROM 90] ROMISZOWSKI A., "Shifting paradigms in education and training : what is the connection with telecommunications ? », Educational and Training Technology International, 27, $n^{\circ} 3,1990$, p. 233-237.

[RUS 95] RUSSEL T. L., The "No significant difference" phenomenon, Université de Caroline du Nord, bureau des télécommunications éducatives, 1995, Rapport de recherche.

[SAB 94] SABA F. \& SHEARER R. L., «Verifying key theoretical concepts in a dynamic model of distance education », The American Journal of Distance Education, $8, n^{\circ} 1$, 1994, p. 36-59.

[SAG 95] SAGA H., "Students' causal attributions of learning from media in distance education », in D. STEWART (Ed.), One world many voices, The Open University, 1995, p. 214-218.

[SAL 77] SALOMON G. \& CLARK R. E., « Reexamining the methodology of research on media an technology in education », Review of Educational Research, 47, $n^{\circ} 1,1977, \mathrm{p}$. 99-120.

[SAL 84] SALOMON G., "Television is "easy" and print is "tough" : the differential investment of mental effort in learning as a function of perceptions and attributions », Journal of Educational Psychology, 76, $n^{\circ} 4,1984$, p. 647-658. 
[SAU 94] SAUVÉ L., « La planification de l'enseignement en formation à distance », in R. VIAU (Ed.), La planification de l'enseignement, deux approches, deux visions ?, Ed. du CRP, 1994, p. 63-90.

[SCH 93] SCHIEMAN E., «Instructional design : beyond heuristics in distance education course development », in G. DAVIES \& B. SAMWAYS (Eds.), Teleteaching, Elsevier, 1993, p. 757-766.

[SCH 96] SCHÖN D. A., « À la recherche d'une nouvelle épistémologie de la pratique et de ce qu'elle implique pour l'éducation des adultes », in J.-M. BARBIER (Ed.), Savoirs théoriques et savoirs d'action, P.U.F., 1996, p. 201-222.

[SEA 82] SEARLE J., Sens et expression, Minuit, 1982.

[SEI 90] SEIGEL A. E. \& DAVIS C., « Delivering undergraduate engineering courses on television : how do grades compare ? , in N. ESTES, J. HEENE \& D. LECLERCQ (Eds.), Proceedings of the 7th International conference on Technology and Education, Vol. 1, 1990, p. 165-167.

[SEL 95] SELLEN A. J., « Remote conversations : the effects of mediating talk with technology », Human-computer interaction, 10, $n^{\circ} 4$, 1995, p. 401-444.

[SHA 81] SHAVELSON R. J. \& STERN P., « Research on teachers' pedagogical thoughts, judgments, decisions, and behavior », Review of Educational Research, 51, $n^{\circ} 4,1981$, p. 455-498.

[SHE 94] SHERRY L., Issues in distance learning, Université de Denver, 1994, rapport de recherche.

[SHO 76] SHORT J., WILLIAMS E. \& CHRISTIE B., The social psychology of telecommunications, Wiley, 1976.

[SIN 75] SINCLAIR J. M. \& COULTHARD R. M., Towards an analysis of discourse, Oxford University Press, 1975.

[STE 88] STEINLE W. J., Telework : present situation and future development of a new form of work organization, North-Holland, 1988.

[STO 90] STONE H. R., « Candid classroom ITV : an evaluation of effectiveness », in N. ESTES, J. HEENE \& D. LECLERCQ (Eds.), Proceedings of the 7th International conference on Technology and Education, Vol. 1, 1990, p. 168-173.

[TAY 95] TAYLOR J. C., JEGEDE O. \& NAIDU S., « Novex analysis : a cognitive science based approach to instructional design for distance education », in D. STEWART (Ed.), One world many voices, The Open University, 1995, p. 466-470.

[TOC 93] TOCHON F. V., L'enseignant expert, Nathan, 1993.

[ULB 95] ULBRICHT K., "Feedback in education and in research on teaching and learning », in R. OLECHOWSKI \& G. KHAN-SVIK (Eds.), Experimental research on teaching and learning, Lang, 1995, p. 32-43.

[WAG 94] WAGNER E. D., "In support of a functional definition of interaction », The American Journal of Distance Education, 8, $n^{\circ} 2$, 1994, p. 6-29.

[WAL 86] WALBERG H. J., « Syntheses of research on teaching », in M. C. WITTROCK (Ed.), Handbook of research on teaching, McMillan, 1986, p. 214-229. 
[WEB 95] WEBB B. R., " Educational research and computer supported co-operative learning », Innovations in Education and Training International, 32, $n^{\circ} 2,1995$, p. 139146.

[WHI 87] WHITTINGTON N., " Is instructional television educationally effective ? A research review », The American Journal of Distance Education, $1, n^{\circ} 1,1987$, p. 4757.

[WOL 95] WOLCOTT L. L., « The distance teacher as reflective practitioner », Educational Technology, 35, $n^{\circ} 1,1995$, p. 39-43.

Philippe Dessus, Benoît Lemaire et Jacques Baillé sont respectivement Attaché temporaire d'enseignement et de recherche en sciences de l'éducation, Maître de conférences en informatique et Professeur en sciences de l'éducation. Au sein de l'équipe DEACT (Didactique expérimentale et appropriation des compétences techniques) du laboratoire des sciences de l'éducation de Grenoble, leurs recherches portent sur l'ergonomie cognitive de la formation. Dans ce cadre, ils poursuivent des travaux sur les situations d'enseignement à distance et sur les environnements informatiques pour l'apprentissage des langues. 